\title{
Neoadjuvant treatment for newly diagnosed advanced ovarian cancer: where do we stand and where are we going?
}

\author{
Michele Moschetta ${ }^{1}$, Stergios Boussios ${ }^{2,3}$, Elie Rassy ${ }^{4,5}$, Eleftherios P. Samartzis ${ }^{6}$, Gabriel Funingana ${ }^{7}$, \\ Mario Uccello ${ }^{8}$ \\ ${ }^{1}$ Cambridge University Hospitals NHS Foundation Trust, Cambridge, UK; ${ }^{2}$ Department of Medical Oncology, Medway NHS Foundation Trust, \\ Gillingham, Kent, UK; ${ }^{3}$ AELIA Organization, 9th Km Thessaloniki-Thermi, Thessaloniki, Greece; ${ }^{4}$ Department of Cancer Medicine, Gustave \\ Roussy Institut, Villejuif, France; ${ }^{5}$ Department of Hematology-Oncology, Hotel Dieu de France University Hospital, Faculty of Medicine, Saint \\ Joseph University, Beirut, Lebanon; ${ }^{6}$ Department of Gynecology, University Hospital Zurich, Frauenklinikstrasse 10, CH-8091, Zurich, Switzerland; \\ ${ }^{7}$ Department of Oncology, University of Cambridge, Cambridge, UK; ${ }^{8}$ Northampton General Hospital NHS Trust, Cliftonville, Northampton, UK \\ Contributions: (I) Conception and design: M Moschetta; (II) Administrative support: None; (III) Provision of study materials or patients: None; (IV) \\ Collection and assembly of data: M Moschetta; (V) Data analysis and interpretation: None; (VI) Manuscript writing: All authors; (VII) Final approval \\ of manuscript: All authors. \\ Correspondence to: Dr. Michele Moschetta, MD, PhD. Medical Oncologist, Cambridge University Hospitals NHS Foundation Trust, Cambridge, UK. \\ Email: michelemoschetta1@gmail.com.
}

\begin{abstract}
Newly diagnosed high grade serous epithelial ovarian cancer (EOC) patients are treated with radical surgery followed by adjuvant platinum and taxane combination chemotherapy. In EOC patients where upfront surgery is contraindicated for medical reasons (e.g., comorbidities or poor performance status), or where complete cytoreduction cannot be achieved, neoadjuvant chemotherapy (NACT) prior to interval debulking surgery (IDS), and adjuvant chemotherapy is an alternative therapeutic option. There is currently a lack of consensus about who are the best candidates to receive NACT, and some authors have even suggested that this approach could be harmful in a subset of patients via promotion of early chemoresistance. Standard and novel imaging techniques together with a better molecular characterization of the disease have the potential to improve selection of patients, but ultimately well designed randomised clinical trials are needed to guide treatment decisions in this setting. The advent of new and effective treatment options (antiangiogenics and PARP inhibitors), now approved for use in the first line and relapse settings has opened the way to clinical trials aiming to investigate these agents as substitute or in addition to chemotherapy in the neoadjuvant setting in molecularly selected EOC patients. Here, we will review the evidence supporting the use of NACT in newly diagnosed EOCs, data highlighting the importance of its use in selected patients, new imaging methodologies and biomarkers that can guide patient selection.
\end{abstract}

Keywords: Neoadjuvant chemotherapy (NACT); epithelial ovarian cancer (EOC); patient selection; imaging; biomarkers

Submitted Feb 17, 2020. Accepted for publication Jun 01, 2020.

doi: 10.21037/atm-20-1683

View this article at: http://dx.doi.org/10.21037/atm-20-1683

\section{Introduction}

High grade serous epithelial ovarian cancer (EOC) of the ovaries, fallopian tubes, and peritoneum is the most common cause of death among women with gynaecologic malignancies and the fifth leading cause of cancer death in women in the
United States (1). High grade serous ovarian cancer is the most common histologic subtype, accounting for over 70\% of all EOCs (2). Three out of four women with EOCs are diagnosed with advanced disease (3), i.e., stage III (disease that has spread throughout the peritoneal cavity or that involves lymph nodes) or stage IV (disease spread to more 
distant sites), and a percentage of them shows resistance to upfront systemic medical treatment (4-6).

The majority of newly diagnosed EOC patients are treated with radical surgery, as initially proposed by Meigs at al. in 1934 and later by Griffiths et al. (7) in 1975, followed by six to eight cycles of adjuvant platinum and taxane combination chemotherapy (1).

Most patients experience disease relapse within the first 5 years despite aggressive treatment at diagnosis, and approximately only a quarter of cases are cured (8).

Three or more cycles of neoadjuvant chemotherapy (NACT) prior to debulking surgery and adjuvant chemotherapy is an alternative option for selected patients (9).

There is currently a lack of consensus about who are the best candidates to receive NACT followed by interval debulking surgery (IDS) and adjuvant chemotherapy (NACT-IDS), and how to best select them. Some authors have even suggested that this approach could be harmful in a subset of patients via promotion of early chemoresistance $(5,6)$. Importantly, NACT offers the opportunity to test upfront chemosensitivity and to identify patients at higher risk of relapse. The advent of new and effective treatment options (antiangiogenics and PARP inhibitors), now approved for use in the first line and relapse settings (1), has opened the way to clinical trials aiming to investigate these agents as substitute or in addition to chemotherapy in the neoadjuvant setting in molecularly selected EOC patients. Neoadjuvant treatment before debulking surgery could also represent an optimal approach for testing new and effective treatments with the potential to accelerate drug development thanks to availability of pre- and posttreatment tumor tissue (9).

Here will review the evidence supporting the use of NACT in newly diagnosed EOCs, data supporting the use of this approach in selected patients, new imaging methodologies and biomarkers that can guide patient selection.

\section{Patient selection: NACT-IDS versus upfront surgery}

The gold standard treatment for management of newly diagnosed EOC patients remains primary debulking surgery followed by platinum and taxane combination adjuvant chemotherapy (1). However, NACT followed by IDS could represent an equally effective and sometimes better tolerated alternative. Complete cytoreduction to no residual disease leads to the best prognosis (10), and it represents the single most important clinical endpoint which has been repeatedly associated with improved survival in patients undergoing debulking surgery.

Initially, the European Organization for Research and Treatment of Cancer (EORTC) - National Cancer Institute of Canada (NCIC) trial randomised newly diagnosed EOCs with stages IIIC or IV ovarian, fallopian tube or primary peritoneal carcinoma to primary debulking surgery followed by adjuvant platinum-based chemotherapy or to NACT with three cycles of platinum-based treatment followed by IDS and adjuvant chemotherapy, if no disease progression was observed (11). Five years later, the CHORUS study, a very similar randomised trial of upfront surgery versus NACT was published (12). Both studies were noninferiority trials and demonstrated equivalent overall survival (OS) in both treatment arms; interestingly, there was less morbidity and mortality in the group receiving NACT versus the group receiving surgery.

These two studies led to broad acceptance of NACT as an equally effective therapy option to the gold standard treatment with upfront radical surgery followed by adjuvant chemotherapy.

The Japanese Clinical Oncology Group (JCOG) 0602 trial again compared upfront radical surgery followed by eight cycles of chemotherapy with four cycles of NACT followed by IDS and four cycles of chemotherapy (13). In this study, the NACT group showed better surgery outcome than the group receiving upfront surgery: it was noted a shorter total surgery time per patient, shorter operation time, less abdominal organ resections, less blood loss during surgery, less requirement of albumin transfusion, and overall, less morbidity after surgery (13).

After these studies were published, the use of NACT gradually increased from 16\% during 2003 to 2010 to 34\% during 2011 to 2012 in stage IIIC disease, and from $41 \%$ to $62 \%$ in stage IV disease (14). However, these studies also opened several questions which will be discussed later in this review; the optimal therapeutic strategy for newly diagnosed EOCs remains still a matter of debate.

In order to achieve complete debulking, more extensive upper abdominal surgical resections are often necessary, including partial pancreatectomy, stripping and/or resection of the diaphragm, partial/total splenectomy, partial liver resection. This implies that surgery in these patients is often a complicated procedure requiring specific surgical training in high volume centres (8). Lack of surgeon's expertise has been identified as important factor for not receiving optimal surgical treatment by $38 \%$ of gynecologic oncologists in 
a survey in Australia and New Zealand (15). Similarly, a low complete debulking rate was highlighted in both the EORTC-NCIC (11) and CHORUS (12) trials questioning the generalizability of these results and highlighting the importance of receiving IDS in specialized centres. The overall clinical conditions and comorbidities of a patient at diagnosis may also limit her chances to receive an optimal extensive debulking surgery. For this reason, it is important to receive an early diagnosis and a prompt treatment to avoid deterioration of clinical conditions.

Considering the importance of disease debulking to no residual disease, NACT could be considered a standard option for a subgroup of women in whom upfront surgery is contraindicated for medical comorbidities, poor performance status or old age, or for those where imaging or laparoscopic staging of the disease suggests that complete cytoreduction is not achievable (9).

Additional attempts to better define the subsets of EOCs patients which could benefit more from NACT than upfront surgery are ongoing $(14,16)$. A more precise and widely accepted definition of unresectability, which would include clinical, imaging, biomarker criteria is urgently needed (17). An exploratory analysis of patients recruited in the EORTC-NCIC trial showed that serum CA-125, age, WHO performance status, tumor grade, tumor histology, the presence of a pelvic mass, or the presence of an omental cake are all not predictive of five-year survival rates independently by the modality of treatment and are not useful criteria to select patients for NACT versus upfront surgery (16).

On the other hand, the size of the largest mass is prognostic for survival following primary surgery; patients carrying the largest tumor mass of a size $<40 \mathrm{~mm}$ were found to have a higher five-year OS rate compared with those with larger tumor sizes (40 versus 14 percent, respectively) if receiving upfront surgery but not if receiving NACT (16). Moreover, women with stage IV disease had higher 5-year OS rates following NACT compared with primary surgery (16). In general, diffuse and/or deep infiltration of the small bowel mesentery, carcinomatosis involving the stomach and/or large parts of the small or large bowel, infiltration of the duodenum and/or parts of the pancreas, involvement of the large vessels of the hepatoduodenal ligament, celiac trunk or behind the porta hepatis, extensive diaphragmatic disease, suprarenal paraaortic lymphadenopathy, multiple liver metastases requiring extended hepatectomy, $>1 \mathrm{~L}$ ascites, extra-peritoneal metastases such as to lung and brain are all considered criteria of surgical unresectability (8).
However, it is important to note that the resectability/ unresectability of the disease ultimately depends on the specific surgical skills and experience of the operator; this has limited the use of universally accepted surgical/imaging criteria for the definition of resectability/unresectability, it has affected the interpretation of results of previous studies in this setting, and it should be an important factor to consider when designing future randomised studies. For example, the ongoing Trial of Radical Upfront Surgical Therapy in advanced ovarian cancer (TRUST), an open, randomized, controlled multi-center trial assessing overall survival after primary cytoreductive surgery versus NACT and subsequent IDS in patients with FIGO stage IIIB-IVB EOC, requires participating centers to fulfil specific quality assurance criteria (i.e., more than $50 \%$ complete resection rate in upfront surgery for FIGO IIIB-IVB patients, at least 36 debulking surgeries per year, etc.). The trial aims to compare overall survival rates after complete resection both in the primary debulking and the NACT group in highly qualified ovarian cancer surgery centers (18).

Additional important questions that remain to be answered are: (I) how to best stage newly diagnosed EOC patients (via imaging, laparoscopy, surgical staging) before deciding treatment approach (upfront surgery or NACTIDS); (II) how to best evaluate the presence of residual tumor after NACT (CT, PET-CT, circulating tumor DNA, or others); (III) how many cycles of NACT are optimal to achieve best outcome; (IV) how to personalize treatment type and treatment duration in patients receiving NACT (9).

Standard and novel imaging techniques together with a better molecular characterization of the disease have the potential to improve selection of patients, but ultimately well designed randomised clinical trials are needed to answer these questions.

\section{Standard and novel methodologies to guide patient selection}

\section{Standard methodologies}

Computed tomography (CT) is used as the standard evaluation method to stage advanced EOC, and assess response to treatment. In patients receiving NACT-IDS, response to treatment via $\mathrm{CT}$ is usually performed after $2-3$ cycles of chemotherapy. CT can only assess size of gross tumor mass or diffuse small nodules but not vitality of the tumor tissue, it lacks of reproducibility and suffers of operator subjectivity (19); changes in tumor size may 
happen after the completion of treatment or never happen even when the tumor is sensitive to treatment. This ultimately means that many patients may receive multiple cycles of ineffective treatment or considered wrongly insensitive to treatment. Alternative methodologies to better assess tumor response are needed (19). Moreover, the availability of methodologies able to assess response to chemotherapy after only one cycle of treatment may rapidly identify insensitive patients and allow them to be promptly switched to a more effective alternative treatment (i.e., surgery); this could allow the development of an adaptive treatment approach based on the specific chemosensitivity of a patient in a more personalized fashion.

It is important to note that the value of identifying early non-responder patients depends on whether this could inform clinical decision making, which includes the use of different treatment options in the same setting; unfortunately, there are currently no alternative neoadjuvant treatments to carboplatin and paclitaxel. The approval of new treatments in the first line setting (1), namely PARP inhibitors, may change this current limitation in the near future and it is likely that these novel agents will be soon tested in the neoadjuvant setting in selected patients with BRCA1/BRCA2 mutations or mutations in other genes of the DNA repair pathways. Moreover, additional novel targeted drugs, namely folate receptor antibodies, vaccines, $\mathrm{T}$ cell therapies, and chimeric antigen receptor $\mathrm{T}$ cells, are also being developed in EOCs with preliminary encouraging results (1); their approvals in the advanced disease may lead to rapid clinical development of these drugs in the neoadjuvant setting.

Finally, a subset of patients (approximately 20\%) with EOC are refractory and progress during NACT (20). Identifying molecular features predictive of response to chemotherapy may further improve patient selection at diagnosis and guide therapeutic decisions.

\section{Positron emission tomography (PET) imaging}

Several studies have assessed whether 18F-FDG-PET imaging and changes in FDG uptake could predict sensitivity to NACT and patient outcome with encouraging results. For example, in a study by Avril et al. (21) there was a significant correlation between OS rate, and the metabolic response after the first and the third cycle of chemotherapy. The median OS for metabolic responders after the first cycle of chemotherapy was 38.3 months, compared with 23.1 months for metabolic non-responders, and after the third cycle, the median OS was 38.9 months for metabolic responders compared with 19.7 months for metabolic non-responders. In another study (22), it was shown that decrease of omental SUVmax after NACT was associated with histopathological response but not PFS. PET imaging could also be useful for guiding decision about the optimal number of cycles of chemotherapy to use before surgery. For example, Martoni et al. (23) found that in patients with complete SUVmax response after 3 cycles, were 3 times more likely to achieve a complete pathological response and no residual disease after debulking surgery compared to those who didn't achieve a complete metabolic response. All these studies suggest that PET imaging could be a useful technique to refine assessment of response to NACT; however further studies are needed to fully define the role of PET imaging in this setting.

\section{Functional MRI imaging}

Functional and advanced MRI techniques, such as diffusionweighted imaging (DWI), dynamic contrast-enhanced (DCE)-MRI and quantitative DCE-MRI histograms (24-27), may also have a role in the assessment of the response to NACT in newly diagnosed advanced EOC patients. These MRI-based techniques can be used to assess changes in tumor tissue components and architecture, tumor stroma and vascularity. For example, a study by Kyriazi et al. (28) found that patients responding to NACT showed a significant change in histogram parameters of apparent diffusion coefficient, an MRI parameter, after the first and third cycle of NACT, but no significant changes were found in patients non-responding to chemotherapy.

Michielsen et al. demonstrated a superiority of whole body (WB)—diffusion-weighted (DWI)/MRI and an almost perfect interobserver agreement compared to CT in the prediction of incomplete resectability (29).

Finally, a recent study by $\mathrm{Li}$ et al. (30) in 56 patients with EOC found that whole solid tumor volume quantitative DCE-MRI histogram parameters could predict the recurrence of EOC and may be potential biomarkers for the prediction of EOC recurrence. All these studies suggest that advanced MRI techniques may be an important imaging tool with the potential to improve patient selection and response assessment in this setting.

\section{Biomarkers}

CA-125 levels correlate with response to chemotherapy 
$(31,32)$, and normalization of the levels of CA-125 prior to IDS are associated with improved survival after IDS (33). Absolute CA-125 levels, and CA-125 regression are known to predict outcome, post-operatively (33); thus CA-125 could be used to identify patients likely to benefit from NACT-IDS early after starting systemic treatment. However, whether early changes of CA-125 levels after one or two cycles of chemotherapy can be an early indicator of response to NACT or can be used to drive decisions regarding the number of cycles of chemotherapy to be used before IDS remains to be investigated in prospective studies. A very recent retrospective analysis carried out in 105 patients with stage III-IV tubo-ovarian cancer treated with NACT found that a reduction of CA-125 levels during NACT, and as early as after the $2^{\text {nd }}$ cycle of chemotherapy, is able to predict successful cytoreductive surgery and long-term clinical outcome in patients with advanced high grade serous and endometrioid ovarian cancer (34), suggesting that CA-125 could be a good biomarker to assess chemosensitivity of EOC patients undergoing NACT.

The degree of response to NACT via histopathological analysis could also provide prognostic information (35-39), and several studies have confirmed the prognostic value of a complete pathological response (pCR) after NACT (36) similarly to what had been shown in breast cancer patients (40).

Several groups have tried to refine histopathological assessment creating composite response scores, incorporating an assessment of necrosis, viable tumor cells, fibrosis, inflammatory changes with conflicting results (41-43).

Importantly, the identification of a NACT response score to risk-stratify patients has the potential to optimize the postoperative management, consideration of maintenance treatment, or participation in clinical trials, with potential of improving long-term outcome.

Circulating tumor free DNA (ctDNA) is emerging as a minimally invasive 'liquid biopsy' tool to guide the use of targeted drugs, and support precision medicine. Circulating tumor DNA-genomic DNA fragments are released into the bloodstream after active secretion via cellular microvesicles or tumor cell lysis (44). Circulating tumor DNA can reflect tumor evolution and the genomic alterations present in primary and/or metastatic tumors. Circulating tumor DNA analysis could allow better molecular characterization and stratification of patients, monitoring of the therapeutic response, and open the opportunity for early intervention independent of detection by imaging modalities or clinical symptoms, and could also help with early identification of cancer, identify mechanisms of resistance to current treatment, and help to select patients likely to benefit from adjuvant treatment after radical surgery (44).

Increased tumor ctDNA it has been shown to be associated with poor survival rate and showed better sensitivity and specificity than CA-125 (45). Based on this evidence, future studies should assess whether early changes of ctDNA levels may be useful in identifying patients who are responding to NACT, whether next generation sequencing of ctDNA could identify patients to be treated with specific molecularly target treatments (i.e., PARP inhibitors), and whether persistence of ctDNA after IDS may identify patients more likely to relapse or likely to require a more intense and prolonged adjuvant treatment.

Homologous recombination (HR) DNA repair pathway is essential to preserve genomic integrity, allowing accurate repair of double-strand DNA breaks, and has been associated with the response to platinum and PARP inhibitor treatments and the overall outcome of patients with EOC (46). Approximately $15-25 \%$ of EOC patients carry germ-line mutations in BRCA1 or BRCA2 genes (47), and these mutations strongly associate with response to PARP inhibitor and platinum chemotherapy (46). Gorodnova et al. (48) proved that EOC patients with BRCA1/2 germ-line mutation show high sensitivity to platinum-based NACT, while Kessous et al. (49) reported that expression of three HR genes (BRCA2, TP53, and FANCB) associated with prolonged OS in EOC patients receiving NACT-IDS. Homologous recombination gene status could be a useful parameter to select patients more likely to benefit from platinum-based NACT or novel treatments like PARP inhibitors, and could guide selection of patients to be enrolled in future neoadjuvant trials.

Recently Lee et al. (50) carried out a detailed analysis of primary tumors and multiple metastatic sites from 30 patients with EOC who received upfront R0 resection (10 patients) versus those who received standard intravenous carboplatin/paclitaxel NACT and achieved either excellent response (10 patients) or poor response (10 patients). Molecular and cellular differences between these clinically defined groups were studied by whole-genome sequencing (WGS), targeted deep sequencing, RNA sequencing (RNAseq), reverse-phase protein array (RPPA), mass spectrometry (MS)-based proteomics and phosphoproteomics, immune profiling, and integrated data analysis of the tumor tissue collected at diagnosis. Significant distinct molecular abnormalities, cellular changes and immune cell repertoire alterations between the groups were identified; these included higher rate of NF1 copy number loss, reduced 
chromothripsis-like patterns, higher levels of strongbinding neoantigens, and a higher number of infiltrated $\mathrm{T}$ cells in the R0 versus the NACT groups. This study opens the way to the identification of new molecular and cellular biomarkers that could be utilised to predict response to NACT and guide treatment decisions in newly diagnosed EOCs; further studies in a more numerous EOC patient population should be carried out in the future to build on these findings.

Finally, immune-biomarkers could also have an important role, and help with the identification of patients to be treated with NACT or with novel immunotherapy agents. Several studies have reported that tumor-infiltrating lymphocytes (TILs) are associated with an improved survival rate in EOC $(51,52)$, not only at diagnosis but also after NACT (53). This was recently confirmed in a retrospective analysis of tumor tissue from 130 patients with EOC; women with higher CD3, PD-L1, and PD-1 expression had improved $\mathrm{OS}(\mathrm{P}=0.03, \mathrm{P}=0.007$, and $\mathrm{P}=0.02$, respectively) (54). Moreover, $\mathrm{PD}-\mathrm{L} 1 / \mathrm{PD}-1$ expression, regardless of being markers for immune suppressive pathways, seemed also to confer a survival benefit (54).

Although, single agent anti-PD1/PDL1 and antiCTLA4 checkpoint inhibitor treatment has shown low response rates in relapsed EOCs (55), subsets of patients have benefitted. Combination phase 2 trials of anti-PD1/ PDL1 checkpoint inhibitors with PARPi in platinum sensitive relapses, and platinum resistance relapses (56) have shown more promising results and are currently being investigated in randomised trials. A randomised phase 2 study investigating the combination of chemotherapy with anti-PDL1 and anti-CTLA4 checkpoint inhibitors in the neoadjuvant setting is currently ongoing and results are expected next year (NCT03249142).

\section{Conclusions}

It is accepted that a subset of newly diagnosed EOC patients may benefit by NACT-IDS, while other patients require upfront surgery to achieve best outcome. However, precise patient selection criteria to guide therapeutic decisions in this setting are currently lacking. Complete surgical debulking with no residual disease remains the most important clinical endpoint that has been repeatedly associated with longer survival. Early diagnosis together with the availability of centralized centres with experience in surgical management of newly diagnosed EOCs are additional factors associated with best outcome. Better understanding of molecular pathogenesis of EOCs, the availability of new targeted treatments (e.g., PARPi) for patients with specific molecular alterations (e.g., BRCA1/2) has opened the way to new trials investigating the use of these agents in the neoadjuvant setting that can potentially change the current treatment paradigm. Novel imaging techniques and biomarkers (e.g., ctDNA) to monitor response to NACT can also open the way to a more personalized and adaptive treatment approach for newly diagnosed EOC patients.

\section{Acknowledgments}

Funding: None.

\section{Footnote}

Provenance and Peer Review: This article was commissioned by the Guest Editors (SB and Nicholas Pavlidis) for the series "Ovarian Cancer: State of the Art and Perspectives of Clinical Research" published in Annals of Translational Medicine. The article was sent for external peer review organized by the Guest Editors and the editorial office.

Conflicts of Interest: All authors have completed the ICMJE uniform disclosure form (available at http://dx.doi. org/10.21037/atm-20-1683). The series "Ovarian Cancer: State of the Art and Perspectives of Clinical Research" was commissioned by the editorial office without any funding or sponsorship. SB served as the unpaid Guest Editors of the series and serves as an unpaid editorial board member of Annals of Translational Medicine from Nov 2019 to Oct 2021. MM is currently a full-time employee of AstraZeneca, Cambridge, UK. The authors have no other conflicts of interest to declare.

Ethical Statement: The authors are accountable for all aspects of the work in ensuring that questions related to the accuracy or integrity of any part of the work are appropriately investigated and resolved.

Open Access Statement: This is an Open Access article distributed in accordance with the Creative Commons Attribution-NonCommercial-NoDerivs 4.0 International License (CC BY-NC-ND 4.0), which permits the noncommercial replication and distribution of the article with the strict proviso that no changes or edits are made and the original work is properly cited (including links to both the 
formal publication through the relevant DOI and the license). See: https://creativecommons.org/licenses/by-nc-nd/4.0/.

\section{References}

1. Lheureux S, Braunstein M, Oza AM. Epithelial ovarian cancer: Evolution of management in the era of precision medicine. CA Cancer J Clin 2019;69:280-304.

2. Prat J. Ovarian carcinomas: five distinct diseases with different origins, genetic alterations, and clinicopathological features. Virchows Arch 2012;460:237-49.

3. Seidman JD, Yemelyanova A, Cosin JA, et al. Survival rates for international federation of gynecology and obstetrics stage III ovarian carcinoma by cell type: a study of 262 unselected patients with uniform pathologic review. Int J Gynecol Cancer 2012;22:367-71.

4. Luo Y, Lee M, Kim HS, et al. Effect of neoadjuvant chemotherapy on platinum resistance in stage IIIC and IV epithelial ovarian cancer. Medicine (Baltimore) 2016;95:e4797.

5. Petrillo M, Ferrandina G, Fagotti A, et al. Timing and pattern of recurrence in ovarian cancer patients with high tumor dissemination treated with primary debulking surgery versus neoadjuvant chemotherapy. Ann Surg Oncol 2013;20:3955-60.

6. Rauh-Hain JA, Nitschmann CC, Worley MJ Jr, et al. Platinum resistance after neoadjuvant chemotherapy compared to primary surgery in patients with advanced epithelial ovarian carcinoma. Gynecol Oncol 2013;129:63-8.

7. Griffiths CT. Surgical resection of tumor bulk in the primary treatment of ovarian carcinoma. Natl Cancer Inst Monogr 1975;42:101-4.

8. Cho JH, Kim S, Song YS. Neoadjuvant chemotherapy in advanced ovarian cancer: optimal patient selection and response evaluation. Chin Clin Oncol 2018;7:58.

9. Leary A, Cowan R, Chi D, et al. Primary Surgery or Neoadjuvant Chemotherapy in Advanced Ovarian Cancer: The Debate Continues. Am Soc Clin Oncol Educ Book 2016;35:153-62.

10. du Bois A, Reuss A, Pujade-Lauraine E, et al. Role of surgical outcome as prognostic factor in advanced epithelial ovarian cancer: a combined exploratory analysis of 3 prospectively randomized phase 3 multicenter trials: by the Arbeitsgemeinschaft Gynaekologische Onkologie Studiengruppe Ovarialkarzinom (AGO-OVAR) and the Groupe d'Investigateurs Nationaux Pour les Etudes des
Cancers de l'Ovaire (GINECO). Cancer 2009;115:1234-44.

11. Vergote I, Trope CG, Amant F, et al. Neoadjuvant chemotherapy or primary surgery in stage IIIC or IV ovarian cancer. N Engl J Med 2010;363:943-53.

12. Kehoe S, Hook J, Nankivell M, et al. Primary chemotherapy versus primary surgery for newly diagnosed advanced ovarian cancer (CHORUS): an open-label, randomised, controlled, non-inferiority trial. Lancet 2015;386:249-57.

13. Onda T, Matsumoto K, Shibata T, et al. Phase III trial of upfront debulking surgery versus neoadjuvant chemotherapy for stage III/IV ovarian, tubal and peritoneal cancers: Japan Clinical Oncology Group Study JCOG0602. Jpn J Clin Oncol 2008;38:74-7.

14. Meyer LA, Cronin AM, Sun CC, et al. Use and Effectiveness of Neoadjuvant Chemotherapy for Treatment of Ovarian Cancer. J Clin Oncol 2016;34:3854-63.

15. Brand AH. Ovarian cancer debulking surgery: a survey of practice in Australia and New Zealand. Int J Gynecol Cancer 2011;21:230-5.

16. van Meurs HS, Tajik P, Hof MH, et al. Which patients benefit most from primary surgery or neoadjuvant chemotherapy in stage IIIC or IV ovarian cancer? An exploratory analysis of the European Organisation for Research and Treatment of Cancer 55971 randomised trial. Eur J Cancer 2013;49:3191-201.

17. Vergote I, du Bois A, Amant F, et al. Neoadjuvant chemotherapy in advanced ovarian cancer: On what do we agree and disagree? Gynecol Oncol 2013;128:6-11.

18. Reuss A, du Bois A, Harter P, et al. TRUST: Trial of Radical Upfront Surgical Therapy in advanced ovarian cancer (ENGOT ov33/AGO-OVAR OP7). Int J Gynecol Cancer 2019;29:1327-31.

19. Rockall A, Munari A, Avril N. New ways of assessing ovarian cancer response: metabolic imaging and beyond. Cancer Imaging 2012;12:310-4.

20. Hacker NF. Neoadjuvant chemotherapy for advanced epithelial ovarian cancer. Who really benefits? Aust N Z J Obstet Gynaecol 2017;57:585-7.

21. Avril N, Sassen S, Schmalfeldt B, et al. Prediction of response to neoadjuvant chemotherapy by sequential F-18-fluorodeoxyglucose positron emission tomography in patients with advanced-stage ovarian cancer. J Clin Oncol 2005;23:7445-53.

22. Vallius T, Peter A, Auranen A, et al. 18F-FDG-PET/CT can identify histopathological non-responders to platinumbased neoadjuvant chemotherapy in advanced epithelial 
ovarian cancer. Gynecol Oncol 2016;140:29-35.

23. Martoni AA, Fanti S, Zamagni C, et al. [18F]FDG-PET/ CT monitoring early identifies advanced ovarian cancer patients who will benefit from prolonged neo-adjuvant chemotherapy. Q J Nucl Med Mol Imaging 2011;55:81-90.

24. Gladwish A, Milosevic M, Fyles A, et al. Association of Apparent Diffusion Coefficient with Disease Recurrence in Patients with Locally Advanced Cervical Cancer Treated with Radical Chemotherapy and Radiation Therapy. Radiology 2016;279:158-66.

25. Hotker AM, Mazaheri Y, Aras O, et al. Assessment of Prostate Cancer Aggressiveness by Use of the Combination of Quantitative DWI and Dynamic Contrast-Enhanced MRI. AJR Am J Roentgenol 2016;206:756-63.

26. Hotker AM, Mazaheri Y, Wibmer A, et al. Use of DWI in the Differentiation of Renal Cortical Tumors. AJR Am J Roentgenol 2016;206:100-5.

27. Just N. Improving tumour heterogeneity MRI assessment with histograms. Br J Cancer 2014;111:2205-13.

28. Kyriazi S, Collins DJ, Messiou C, et al. Metastatic ovarian and primary peritoneal cancer: assessing chemotherapy response with diffusion-weighted MR imaging--value of histogram analysis of apparent diffusion coefficients. Radiology 2011;261:182-92.

29. Michielsen K, Dresen R, Vanslembrouck R, et al. Diagnostic value of whole body diffusion-weighted MRI compared to computed tomography for pre-operative assessment of patients suspected for ovarian cancer. Eur J Cancer 2017;83:88-98.

30. Li HM, Tang W, Feng F, et al. Whole solid tumor volume histogram parameters for predicting the recurrence in patients with epithelial ovarian carcinoma: a feasibility study on quantitative DCE-MRI. Acta Radiol 2020;61:1266-76.

31. Rustin GJ, Nelstrop AE, McClean P, et al. Defining response of ovarian carcinoma to initial chemotherapy according to serum CA 125. J Clin Oncol 1996;14:1545-51.

32. Crawford SM, Peace J. Does the nadir CA125 concentration predict a long-term outcome after chemotherapy for carcinoma of the ovary? Ann Oncol 2005;16:47-50.

33. Vasudev NS, Trigonis I, Cairns DA, et al. The prognostic and predictive value of CA-125 regression during neoadjuvant chemotherapy for advanced ovarian or primary peritoneal carcinoma. Arch Gynecol Obstet 2011;284:221-7.

34. Kessous R, Wissing MD, Piedimonte S, et al. CA-125 reduction during neoadjuvant chemotherapy is associated with success of cytoreductive surgery and outcome of patients with advanced high-grade ovarian cancer. Acta Obstet Gynecol Scand 2020;99:933-40.

35. Ferron JG, Uzan C, Rey A, et al. Histological response is not a prognostic factor after neoadjuvant chemotherapy in advanced-stage ovarian cancer with no residual disease. Eur J Obstet Gynecol Reprod Biol 2009;147:101-5.

36. Petrillo M, Zannoni GF, Tortorella L, et al. Prognostic role and predictors of complete pathologic response to neoadjuvant chemotherapy in primary unresectable ovarian cancer. Am J Obstet Gynecol 2014;211:632.e1-8.

37. Le T, Shahriari P, Hopkins L, et al. Prognostic significance of tumor necrosis in ovarian cancer patients treated with neoadjuvant chemotherapy and interval surgical debulking. Int J Gynecol Cancer 2006;16:986-90.

38. Muraji M, Sudo T, Iwasaki S, et al. Histopathology predicts clinical outcome in advanced epithelial ovarian cancer patients treated with neoadjuvant chemotherapy and debulking surgery. Gynecol Oncol 2013;131:531-4.

39. Le T, Williams K, Senterman M, et al. Omental chemotherapy effects as a prognostic factor in ovarian cancer patients treated with neoadjuvant chemotherapy and delayed primary surgical debulking. Ann Surg Oncol 2007;14:2649-53.

40. Liedtke C, Mazouni C, Hess KR, et al. Response to neoadjuvant therapy and long-term survival in patients with triple-negative breast cancer. J Clin Oncol 2008;26:1275-81.

41. Sassen S, Schmalfeldt B, Avril N, et al. Histopathologic assessment of tumor regression after neoadjuvant chemotherapy in advanced-stage ovarian cancer. Hum Pathol 2007;38:926-34.

42. Le T, Alshaikh G, Hopkins L, et al. Prognostic significance of postoperative morbidities in patients with advanced epithelial ovarian cancer treated with neoadjuvant chemotherapy and delayed primary surgical debulking. Ann Surg Oncol 2006;13:1711-6.

43. Bohm S, Faruqi A, Said I, et al. Chemotherapy Response Score: Development and Validation of a System to Quantify Histopathologic Response to Neoadjuvant Chemotherapy in Tubo-Ovarian High-Grade Serous Carcinoma. J Clin Oncol 2015;33:2457-63.

44. Oliveira KCS, Ramos IB, Silva JMC, et al. Current perspectives on circulating tumor DNA, precision medicine, and personalized clinical management of cancer. Mol Cancer Res 2020;18:517-28.

45. Kamat AA, Baldwin M, Urbauer D, et al. Plasma cellfree DNA in ovarian cancer: an independent prognostic 
biomarker. Cancer 2010;116:1918-25.

46. Boussios S, Karathanasi A, Cooke D, et al. PARP Inhibitors in Ovarian Cancer: The Route to "Ithaca". Diagnostics (Basel) 2019;9:55.

47. Boussios S, Karihtala P, Moschetta M, et al. Combined Strategies with Poly (ADP-Ribose) Polymerase (PARP) Inhibitors for the Treatment of Ovarian Cancer: A Literature Review. Diagnostics (Basel) 2019;9:55.

48. Gorodnova TV, Sokolenko AP, Ivantsov AO, et al. High response rates to neoadjuvant platinum-based therapy in ovarian cancer patients carrying germ-line BRCA mutation. Cancer Lett 2015;369:363-7.

49. Kessous R, Octeau D, Klein K, et al. Distinct homologous recombination gene expression profiles after neoadjuvant chemotherapy associated with clinical outcome in patients with ovarian cancer. Gynecol Oncol 2018;148:553-8.

50. Lee S, Zhao L, Rojas C, et al. Molecular Analysis of Clinically Defined Subsets of High-Grade Serous Ovarian Cancer. Cell Rep 2020;31:107502.

51. Zhang L, Conejo-Garcia JR, Katsaros D, et al. Intratumoral $\mathrm{T}$ cells, recurrence, and survival in epithelial ovarian cancer. N Engl J Med 2003;348:203-13.

Cite this article as: Moschetta M, Boussios S, Rassy E, Samartzis EP, Funingana G, Uccello M. Neoadjuvant treatment for newly diagnosed advanced ovarian cancer: where do we stand and where are we going? Ann Transl Med 2020;8(24):1710. doi: 10.21037/atm-20-1683
52. Hwang WT, Adams SF, Tahirovic E, et al. Prognostic significance of tumor-infiltrating T cells in ovarian cancer: a meta-analysis. Gynecol Oncol 2012;124:192-8.

53. Mesnage SJL, Auguste A, Genestie C, et al. Neoadjuvant chemotherapy (NACT) increases immune infiltration and programmed death-ligand 1 (PD-L1) expression in epithelial ovarian cancer (EOC). Ann Oncol 2017;28:651-7.

54. Martin de la Fuente L, Westbom-Fremer S, Arildsen NS, et al. PD-1/PD-L1 expression and tumor-infiltrating lymphocytes are prognostically favorable in advanced high-grade serous ovarian carcinoma. Virchows Arch 2020;477:83-91.

55. Gonzalez-Martin A, Sanchez-Lorenzo L. Immunotherapy with checkpoint inhibitors in patients with ovarian cancer: Still promising? Cancer 2019;125 Suppl 24:4616-22.

56. Konstantinopoulos PA, Waggoner S, Vidal GA, et al. Single-Arm Phases 1 and 2 Trial of Niraparib in Combination With Pembrolizumab in Patients With Recurrent Platinum-Resistant Ovarian Carcinoma. JAMA Oncol 2019;5:1141-9. 